\title{
Spatio-temporal image correlation (STIC) in evaluation of advanced neuroendocrine tumours
}

\author{
Ksenia Janas' ${ }^{1}$, Marek J. Kudła ${ }^{2}$, Adam Janas ${ }^{3,4}$, Jolanta Blicharz-Dorniak', Beata Kos-Kudła ${ }^{5}$ \\ ${ }^{1}$ Department of Endocrinology and Neuroendocrine Tumors, Medical University of Silesia, Katowice, Poland \\ ${ }^{2}$ Clinical Department of Perinatology and Oncological Gynecology, School of Health Sciences in Katowice, Medical University \\ of Silesia, Katowice, Poland \\ ${ }^{3}$ Center of Cardiovascular Research and Development, American Heart of Poland, Katowice, Poland \\ ${ }^{4}$ Faculty of Medicine and Health Sciences, Andrzej Frycz Modrzewski Krakow University, Kraków, Poland \\ ${ }^{5}$ Department of Endocrinology and Neuroendocrine Tumors, Department of Pathophysiology and Endocrinology, Medical University \\ of Silesia, Katowice, Poland
}

\begin{abstract}
Introduction: 4D Ultrasound technology merging the Power Doppler option called High-Definition Flow (HDF) with Spatio-Temporal Image Correlation (STIC) is used in gynaecology and obstetrics. It seems to be a promising tool in assessing tissue vascularisation. The aim of the paper was to assess whether HDF STIC technique could be a useful tool for the evaluation of gastro-entero-pancreatic neuroendocrine neoplasm (GEP-NEN) advancement.

Material and methods: Forty-eight patients [mean age $57.7 \pm 10.3$ years; male 40.9\% ( $\mathrm{n}=18)$ ] diagnosed with metastatic GEP-NENs were included in the analysis. All subjects were enrolled in the Department of Endocrinology and Neuroendocrine Tumours, Medical University of Silesia, Katowice. We analysed liver metastases. Patients with G3 grading or faintly vascularised lesions were excluded. HDF STIC volumes were acquired during USG examination. 4D indices [volumetric pulsatility index (vPI) and volumetric systolic/diastolic index (vS/D)] were calculated using dedicated software.

Results: There were no correlations between vS/D and Ki-67 [p = 0.67; $r=-0.19 ;(-0.28-0.19)], \mathrm{CgA}[\mathrm{p}=0.47 ; \mathrm{r}=-0.11 ;(-0.38-0,19)]$, 5-HIAA [ $\mathrm{p}=0.52 ; \mathrm{r}=-0.09(-0.37-0.2)]$, serotonin $[\mathrm{p}=0.83 ; \mathrm{r}=-0.03(-0.32-0.26)]$, and VCAM-1 $(\mathrm{p}=0.62 ; \mathrm{r}=0.09(-03-0.47)]$. The were no correlations between vPI and Ki-67 [ $p=0.29 ; \mathrm{r}=-0.16(-0.45-0.14)], \mathrm{CgA}(\mathrm{p}=0.46 ; \mathrm{r}=-0.11(-0.39-0.19)], 5-\mathrm{HIAA}[\mathrm{p}=0.52 ; \mathrm{r}=-0.09$ $(-0.37-0.2)]$, serotonin $[p=0.82 ; r=-0.03(-0.32-0.26)]$, and VCAM- $1(p=0.62 ; r=-0.09(-03-0.47)]$. There was no significant difference between carcinoid versus non-carcinoid patients if compared by vS/D and vPI ( $p=0.62, p=0.61$, respectively).

Conclusions: HDF STIC seems not to be an efficient marker to assess advancement of NENs, due to lack of correlation with widely used and approved markers of progression. (Endokrynol Pol 2019; 70 (3): 219-223)
\end{abstract}

Key words: NENs; metastasis; HDF; STIC; four-dimensional sonography

\section{Introduction}

Neuroendocrine neoplasms (NENs) are a heterogeneous group of relatively rare neoplasms developing from diffuse endocrine system cells. About $70 \%$ of all NENs are gastro-entero-pancreatic neuroendocrine neoplasms (GEP-NENs), which constitute approximately $2 \%$ of all gastrointestinal tract neoplasms. NENs could produce hormones, peptides, and biogenic amines. Predominantly the amount of those substances is insufficient to present clinical symptoms (non-functional NENs) [1]. Over $50 \%$ of GEP-NENs are metastatic at diagnosis [1-3]. 65-95\% of those tumours show hepatic metastasis [3].

Transabdominal ultrasonography, due to its wide availability and low cost, is generally the first imaging technique performed in diagnosing abdominal disorders. The sensitivity of the exam is determined by the lesion location, anatomical and technical conditions, as well as the physician's experience [1].

Characteristic ultrasonography features of neuroendocrine liver metastases are mixed hyper/hypoechoic pattern with central cystic areas and hyper-vascularisation in colour/power Doppler imaging [1, 3].

High-definition flow (HDF) is a recent advance in Doppler technology. This technique is more sensitive to slow-flow small vessels than other Doppler techniques [4-5]. New software allows the calculation of three-dimensional (3D) indices using HDF imaging [6]. Spatiotemporal image correlation (STIC) enables the acquisition of gated single cardiac cycle in a four-dimentional (4D) technique, obtaining data in space and time $[4,7]$. This technique is routinely used in obstetrics and gynaecology diagnostics. Diagnosis of NENs is expensive and time-consuming; therefore, 
in this study, we would like to assess whether STIC technique could be a useful tool for evaluation of NEN advancement.

\section{Material and methods}

\section{Subject}

Sixty-two patients were enrolled in this prospective study. All subjects enrolled in the Department of Endocrinology and Neuroendocrine Tumours, Medical University of Silesia, Katowice were diagnosed with GEP-NEN or NEN of unknown primary site with liver metastases. In all patients, liver metastases were confirmed in non-invasive imaging tests like ultrasonography, magnetic resonance, computed tomography, and positron emission tomography. Moreover, in all cases NEN diagnosis was confirmed in histopathological examination performed by an experienced pathomorphologist. Only subjects with NEN grading G1 or G2 were included. Patients with faintly vascularised liver metastases and those diagnosed with NEN G3 were excluded from analysis. What is more, none of the patients included in the study was diagnosed with any other type of cancer.

\section{Procedural characteristic}

Ultrasonography (US) and Doppler ultrasonography imaging (DUS) of the liver were performed in all patients included in this study. Ultrasonography was performed by single physician with 10 years' experience in abdominal US using the Voluson E8 Expert System (GE Healthcare) equipped with a 3D/4D Matrix Convex Probe (GE RM6C). If many metastases were found in the liver, the most vascularised one was chosen for analysis.

The sonographic protocol and volume analysis have been reported elsewhere [5-8]. The two-dimensional HDF gate was activated and superimposed over the dominant metastases to assess the highest vascularisation area. The long axis of the transducer (i.e. the main $y$-axis on the sonographic screen) was then directed to this point while collecting the sweep. Subsequently, STIC was activated, and a 4D STIC-HDF volume was acquired from the patient (who was asked to remain as still as possible) and then saved for further evaluation. Volume acquisition was repeated when flash-type artefacts appeared due to respiratory movements.

The 3D volume size of the region of interest was set up in the system as constant to cover identical size volumes for all the cases. The HDF Doppler settings were set as follows: $\mathrm{HDF}$ window angle, $70^{\circ}$; zoom, 1.4; depth of the HDF window, $40 \mathrm{~mm}$; pulse repetition frequency, $0.3 \mathrm{kHz}$; gain, 0.2 ; frequency, mid; HDF quality, normal; wall motion filter, low 1 ; smooth, 3/5; ensemble, 10; flow resolution, mid 2; line density, 9; balance, greater than 180; and HD map, 1 . The HDF refreshing frequency resulting from such settings was $15 \mathrm{~Hz}$. The STIC sweep angle was $40^{\circ}$, and the acquisition time was 15 seconds. 4D STIC-HDF analyses were performed using dedicated software - 4DView GE Healthcare Version 10.2. First a chain of 3D HDF volumes forming the STIC loop was activated to choose the moment of highest and lowest vascularisation of the liver lesion. The point of highest vascularisation was brought into the centre of the screen (on the y-axis), and each volume of the chain was saved as $3 \mathrm{D}$ record. A sphere volume of $1 \mathrm{~cm}^{3}$ was set by the operator. After accepting the volume size, HDF histograms were calculated automatically. Two 3D volumes with VI (vascularisation index) values were picked - the highest for systole and the lowest for diastole. Values were saved for further analysis.

All patients underwent a complete biochemical evaluation, including serum levels of chromogranin $\mathrm{A}(\mathrm{CgA})$, serotonin, and vascular cell adhesion molecule-1 (VCAM-1), as well as carcinoid syndrome screening including daily urinary excretion of 5-hydroxyindoleacetic acid (5-HIAA). Blood and urine specimens were collected during hospitalisations when metastases in the liver were discovered. Assessment of histological malignancy was based on Ki-67 prolifera- tion index, determined by immunohistochemical analysis. NEN G1 was defined as $\mathrm{Ki}-67<3 \%$, G2 3-20\%, and G3 > 20\%.

\section{Study definitions}

Using the obtained vascularisation index (VI) two 4D ratios were calculated: the volumetric pulsatility index: $v P I=$ (systolic VI - diastolic VI) / mean VI and the volumetric systolic/diastolic index: $v S / D=$ systolic VI / diastolic VI.

\section{Safety and ethics}

This prospective nonrandomised observational study was conducted with standard ethical guidelines. The agreement of the local Bioethics Committee was obtained before any study procedure.

\section{Statistical analysis}

Continuous variables are presented as mean \pm SD or median (IRQ). Data were compared using Student's t-test for parametric and UMann Whitney test for non-parametric continuous variables. For correlation assessment Pearson test was used for parametric and Spearman test for nonparametric variables. Categorical variables are reported as frequencies (percentage). All reported P-values are two-tailed, and $p<0.05$ was considered significant. GraphPad 6 Prism was used for statistical analysis.

\section{Results}

Sixty-two patients were enrolled in the study. After histopathological analysis seven subjects were excluded due to G3 grading. The remaining patients had USG with DUS performed, and the subsequent seven patients were excluded from analysis due to faintly vascularised metastases. Forty-eight patients were included to STIC-HDF analysis.

Mean patient age was $57.7 \pm 10.3$ years, and $40.9 \%$ $(\mathrm{n}=18)$ were male. The mean body mass index was $24.1 \pm$ 4.7. Patients' demographic data are shown in Table I. In USG assessment, the mean vertical and horizontal diameters of metastases chosen for STIC evaluation were $36.9 \pm 19.8 \mathrm{~mm}$ and $36.1 \pm 21.5 \mathrm{~mm}$, respectively.

The pancreas was the most common primary tumour site (PTS) - 39.6\% ( $\mathrm{n}=19)$, and second most common PTS was the ileocecal valve and small intestine.

Table I. Patients' demographic data

\begin{tabular}{lc}
\hline Demographic characteristic & $\%(\mathbf{n})$ \\
\hline Diabetes & $29.2 \%(14)$ \\
\hline Hypertension & $54.2 \%(26)$ \\
\hline Dyslipidaemia & $39.6 \%(19)$ \\
\hline Any thyroid dysfunction & $79.2 \%(38)$ \\
\hline Other endocrinology disease & $29.2 \%(14)$ \\
\hline Coronary Artery Disease (CAD) & $6.3 \%(3)$ \\
\hline Anaemia & $43.8 \%(21)$ \\
\hline Renal impairment & $25 \%(12)$ \\
\hline
\end{tabular}


Table II. Primary tumour site location

\begin{tabular}{lc}
\hline Primary tumour site & $\%(\mathbf{n})$ \\
\hline Pancreas & $39.6 \%(19)$ \\
\hline lleocecal valve & $12.5 \%(6)$ \\
\hline Small intestine & $12.5 \%(6)$ \\
\hline Rectum & $2.1 \%(1)$ \\
\hline Stomach & $4.2 \%(2)$ \\
\hline Ampulla of Vater & $2.1 \%(1)$ \\
\hline Unknown & $27.1 \%(13)$ \\
\hline
\end{tabular}

The detailed PTS are shown in Table II. The mean vS/D was $1.25 \pm 2.71$, whereas the mean vPI was $0.28 \pm 0.28$.

There were no correlations between vS/D and Ki-67 [p $=0.67 ; \mathrm{r}=-0.19 ;(-0.28-0.19)], \mathrm{CgA}[\mathrm{p}=0.47$; $\mathrm{r}=-0.11 ;(-0.38-0,19)], 5$-HIAA [p $=0.52 ; \mathrm{r}=-0.09$ $(-0.37-0.2)]$, serotonin $[\mathrm{p}=0.83 ; \mathrm{r}=-0.03(-0.32-0.26)]$, and VCAM-1 $[p=0.62 ; r=0.09(-03-0.47)]$ (Fig. 1). The were no correlations between vPI and $\mathrm{Ki}-67[\mathrm{p}=0.29$; $\mathrm{r}=-0.16(-0.45-0.14)], \mathrm{CgA}[\mathrm{p}=0.46 ; \mathrm{r}=-0.11$ $(-0.39-0.19)], 5-H I A A$ [ $p=0.52, r=-0.09(-0.37-0.2)]$, serotonin $[\mathrm{p}=0.82 ; \mathrm{r}=-0.03(-0.32-0.26)]$, and VCAM-1 $[p=0.62 ; r=-0.09(-03-0.47)]$ (Fig. 2). Moreover, there were no significant differences between carcinoid versus non-carcinoid patients if compared by vS/D and vPI $p=0.62$ and $p=0.61$, respectively (Fig. 3).

\section{Discussion}

This current prospective study reports the assessment of STIC technique for evaluation of neuroendocrine liver metastases. To the best of our knowledge, the present study, for the first time, describes an attempt to use STIC in the evaluation of NEN advancement. In this single-arm, prospective study the STIC did not show any correlation with Ki-67 and other biomarkers of NENs, like chromogranin A, 5-HIAA, serotonin, and VCAM-1. Moreover, there were no differences in vS/D and vPI between patients with functional and non-functional NENs. It is worth noting that $12.7 \%$ (7) of patients had metastases that could not be assessed in STIC due to faint vascularity.

STIC technology is widely used in gynaecology and obstetrics. It is an efficient method in 4D foetal echocardiography used for prenatal screening of heart defects [9]. The 3D sampling technology used in our study was previously described as a promising method for evaluation of ovarian tumours. In a study in which 27

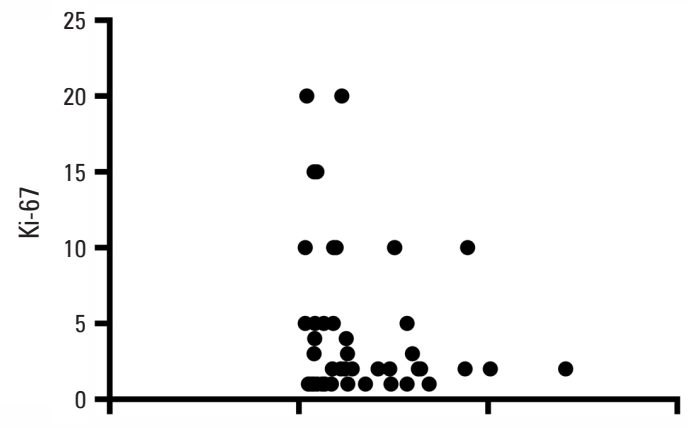

$\mathrm{vS} / \mathrm{D}$

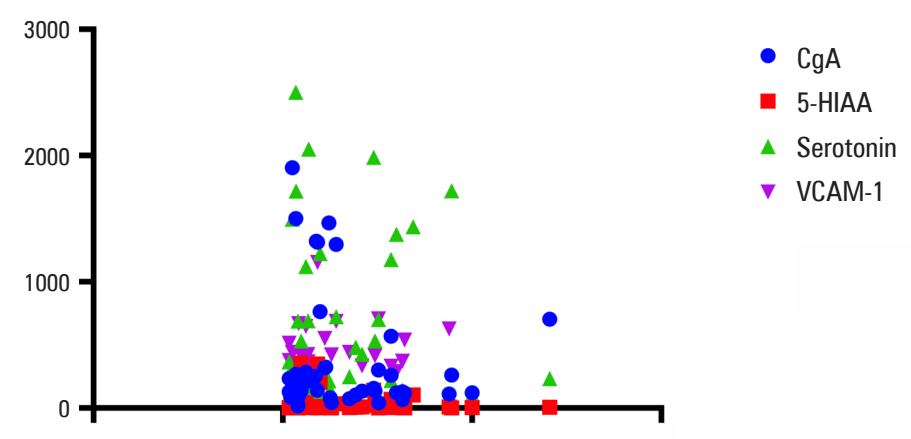

vS/D

Figure 1. Graph of correlation between volumetric systolic/diastolic index (vS/D) vs. Ki-67 and other biochemical markers

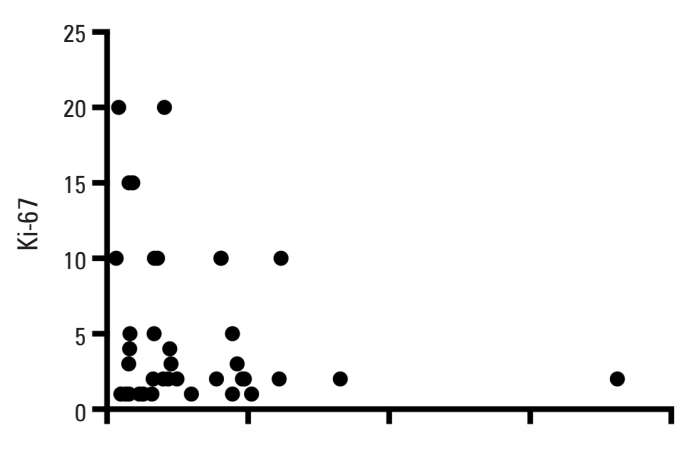

vS/D

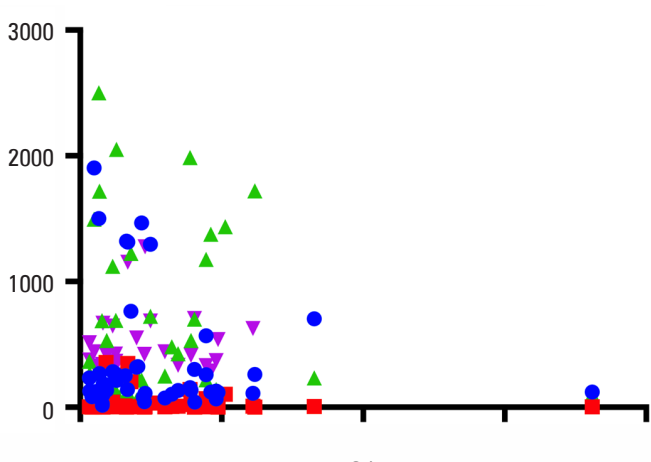

vS/D

Figure 2. Graph of correlation between volumetric pulsatility index (vPI) vs. Ki-67 and other biochemical markers 

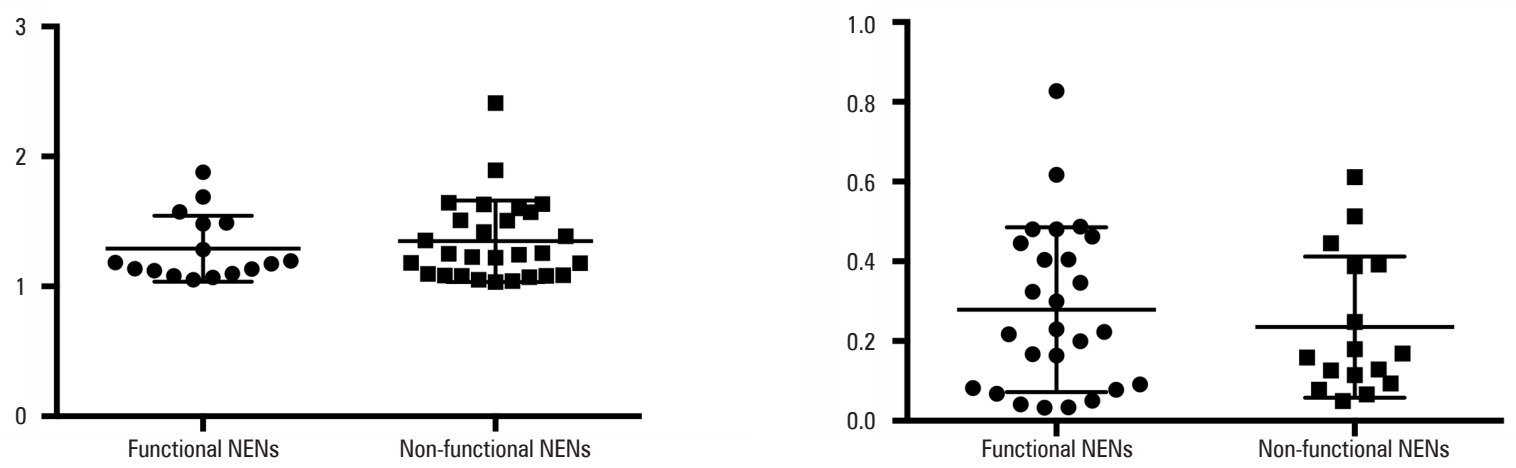

Figure 3. Graph of correlation between volumetric systolic/diastolic index (vS/D) vs. functional and non-functional neuroendocrine neoplasms (NENs) and volumetric pulsatility index (vPI) vs. functional and non-functional NENs

cases of ovarian tumours were analysed, and 53 healthy women formed the control group, a virtual spherical tissue sample of $1 \mathrm{~cm}^{2}$ was taken from the place of highest vessel density contained within the contours of the gland. Spherical sampling with 3D ultrasound power Doppler angiography achieved a higher degree of discrimination between the groups compared to the whole solid-part approach. The authors presumed that this is a sensitive approach to differentiate between tumour and healthy tissue [10].

Recently Polanski et al. tried to assess whether STIC vascularity indices correlate with assisted reproductive treatment outcomes. The authors concluded that this method is not a useful tool to predict outcomes of the mentioned treatment; however, it seemed to be more accurate in predicting first-trimester pregnancy loss [11].

Kim et al. compared HDF Doppler technique with colour Doppler ultrasonography and power Doppler ultrasonography for assessment of hepatic vasculature in native and transplanted liver. Their study revealed that HDF technique provides better resolution to depict hepatic vessels and improve sensitivity to small vessels [4].

Kudla and Alcazar proved that STIC combined with HDF is a useful tool for assessing ovarian vascularisation [5-8]. The first study affirmed that $3 \mathrm{D}$ vascular indices are higher when calculated using HFD than when using conventional power Doppler (PD). The authors concluded that HDF technique is more sensitive than PD in obtaining Doppler signals [7]. The next paper showed that the STIC-HDF method allows evaluation of $3 \mathrm{D}$ vascular indices in the cardiac cycle, and the vascularisation index (VI) depends on the moment of cardiac cycle whereas the flow index (FI) seemed to be independent of the cardiac cycle [5]. Another analysis pointed out that assessment of ovarian vascularisation using STIC-HDF spherical sampling is reliable. The authors introduced also new 4D vascular indices [6].
The recent study showed that calculating the mean VI using entire STIC sequence is as valuable as calculating VI choosing the highest and lowest colour signals determined by the operator [8].

Data about use of the STIC-HDF method are limited. In the available source literature this method seems to be a promising tool for assessing tissue vascularisation. Diagnosing NENs requires expensive and advanced imaging methods. Therefore, in our study we tried to find an accessible and cost-effective method for neuroendocrine liver metastases assessment. However, according to our results, this method seems to have limited usage for evaluation of disease advancement.

\section{Study limitations}

The main limitation of this study is a lack of comparison STIC in patients with different progression free-survival. Moreover, the choice of most vascularised metastases was done subjectively by an ultrasonographer.

One of the basic limitations of using this method in the case of metastasis of neuroendocrine tumors in the liver turned out to be Power Doppler sensitivity to tissue movements. Our results of the Power Doppler STIC indices in the liver in comparison to previous ovarian observations are burdened with low vascularisation of the neuroendocrine metastatic tumors and an error due to artifacts originating from the heart and large vessel movements due to their close location.

\section{Conclusions}

In this hypothesis-generating prospective study, STIC was not an efficient marker for assessment of advancement of NENs, due to lack of correlation with widely used and approved markers of progression.

\section{Funding}

The study was supported by Medical University of Silesia - project no. KWN-1-178/N/6/K. 


\section{References}

1. Kos-Kudła B, Blicharz-Dorniak J, StrzelczykJ. Diagnostic and therapeutic guidelines for gastro-entero-pancreatic neuroendocrine neoplasms (recommended by the Polish Network of Neuroendocrine Tumours). Endokrynol Pol. 2017; 68(2): 79-110, doi: 10.5603/EP.2017.0015, indexed in Pubmed: 28597909.

2. Pavel M, O'Toole $\mathrm{D}$, Costa $\mathrm{F}$, et al. Vienna Consensus Conference participants. ENETS Consensus Guidelines Update for the Management of Distant Metastatic Disease of Intestinal, Pancreatic, Bronchial Neuroendocrine Neoplasms (NEN) and NEN of Unknown Primary Site. Neuroendocrinology. 2016; 103(2): 172-185, doi: 10.1159/000443167, indexed in Pubmed: 26731013.

3. Frilling A, Modlin IM, Kidd M, et al. Working Group on Neuroendocrine Liver Metastases. Recommendations for management of patients with neuroendocrine liver metastases. Lancet Oncol. 2014; 15(1): e8-e21, doi: 10.1016/S1470-2045(13)70362-0, indexed in Pubmed: 24384494.

4. Kim SeH, Lee JM, Kim YJ, et al. High-definition flow Doppler ultrasonographic technique to assess hepatic vasculature compared with color or power Doppler ultrasonography: preliminary experience. J Ultrasound Med. 2008; 27(10): 1491-1501, indexed in Pubmed: 18809960.

5. Kudla MJ, Alcázar JL. Spatiotemporal image correlation using high-definition flow: a new method for assessing ovarian vascularization. J Ultrasound Med. 2010; 29(10): 1469-1474, indexed in Pubmed: 20876901.

6. Kudla MJ, Alcázar JL. Spatiotemporal image correlation with spherica sampling and high-definition flow: new 4-dimensional method for assessment of tissue vascularization changes during the cardiac cycle: reproducibility analysis. J Ultrasound Med. 2012; 31(1): 73-80, indexed in Pubmed: 22215772

7. Alcázar JL, Kudla MJ. Three-dimensional vascular indices calculated using conventional power Doppler and high-definition flow imaging. are there differences? J Ultrasound Med. 2010; 29(5): 761-766, indexed in Pubmed: 20427788.

8. Kudla MJ, Kandzia T, Alcázar JL. Comparison of two methods for calculating the mean vascularization index of ovarian stroma on the basis of spatio-temporal image correlation high-definition flow technology. Ultrasound Med Biol. 2013; 39(11): 2202-2204, doi: 10.1016/j.ultrasmedbio.2013.05.010, indexed in Pubmed: 23969164.

9. Viñals F, Poblete P, Giuliano A. Spatio-temporal image correlation (STIC) a new tool for the prenatal screening of congenital heart defects. Ultrasound Obstet Gynecol. 2003; 22(4): 388-394, doi: 10.1002/uog.883, indexed in Pubmed: 14528475.

10. Kudla MJ, Timor-Tritsch IE, Hope JM, et al. Spherical tissue sampling in 3-dimensional power Doppler angiography: a new approach for evaluation of ovarian tumors. J Ultrasound Med. 2008; 27(3): 425-433 doi: 10.7863/jum.2008.27.3.425, indexed in Pubmed: 18314521.

11. Polanski LT, Baumgarten MN, Brosens J, et al. Endometrial spatio-temporal image correlation (STIC) and prediction of outcome following assisted reproductive treatment. Eur J Obstet Gynecol Reprod Biol. 2016; 203: 320-325, doi: 10.1016/j.ejogrb.2016.06.015, indexed in Pubmed: 27423530. 